\title{
Sliding repetitive control with adaptive bound estimation for motion systems liable to periodic exogenous signals
}

\author{
Yu-Sheng Lu*, Yueh-Tsang Li** and Ming-Chang Lin*** \\ *Dept. of Mechatronic Engineering, National Taiwan Normal Univ., Taipei, Taiwan \\ **Dept. of Mechanical Engineering, National Yunlin Univ. of Sci. \& Tech., Yunlin, Taiwan \\ ***Dept. of Mechanical Engineering, Chung Yuan Christian Univ., Taoyuan, Taiwan \\ *Corresponding Author: luys@ntnu.edu.tw
}

Submitted: 17/10/2019

Revised: $01 / 10 / 2020$

Accepted: 08/10/2020

\begin{abstract}
Periodic exogenous signals often exist in motion systems, especially those involving one or more rotating elements. These periodic exogenous signals deteriorate the performance of motion systems, and these adverse effects cannot be practically eliminated by straightforwardly increasing feedback control gains due to sensor noise, actuator saturation, and unmodeled plant dynamics. This paper describes a sliding repetitive controller for motion systems subject to periodic exogenous signals. Moreover, an adaptive law for bound estimation is devised to ensure the presence of a sliding motion for both repetitive learning and disturbance observation. The tracking motion system of a disk drive is considered in practice, and a traditional repetitive controller is also implemented for performance comparisons with the proposed scheme. Experimental results are reported in this paper, showing the efficacy of the proposed scheme.
\end{abstract}

Keywords: Disturbance observer; Motion system; Periodic exogenous signal; Repetitive control; Sliding mode.

\section{INTRODUCTION}

Mechanical motion systems are often subject to periodic exogenous signals. For a four-bar linkage, such as a crank slide or a slotted link mechanism, there exists a periodic disturbance to its input shaft that drives at a constant rotational speed. Another example is a disk drive, in which the data track's trajectory to be followed has significant repeatable runout (Gu et al., 2014), mostly due to disk and spindle eccentricity. The control problem with such mechanical systems is challenging due to the persistent existence of periodic exogenous signals. A straightforward approach to dealing with this control problem is to use a high-gain control strategy by increasing controllers' gains as much as possible. However, the controllers' gains are practically restricted by unmodeled dynamics and sensor noises and, hence, cannot be increased indefinitely, leading to limited improvement of tracking performance.

Repetitive control is an effectual design tool for plants subject to periodic exogenous signals (Alsubaie \& Rogers, 2019; Jiangand \& Hong, 2019). It has been applied to many practical systems, such as disk drives (Chen et al., 2008), robot manipulators (Fateh et al., 2013; Oliveira \& Lages, 2016), electrohydraulic systems (Yang et al., 2018), vibration control (Hillerstrom, 1996), and switched boost converters (Ma et al., 2019). Its effectiveness on dealing with periodic exogenous signals has been well explained using the internal model principle (Xu et al., 2017). It ensures excellent tracking of a periodic command input or rejection of a periodic external disturbance asymptotically, rather than in the transient state. On the other hand, the sliding control approach (Kara \& Mary, 2018) is a nonlinear strategy that can robustly give excellent transient performance in the existence of system perturbations. However, it 
is unable to be completely insensitive to periodic exogenous disturbances, and there could exist steady-state errors due to the exogenous disturbances. In the study (Lu \& Wang, 2009), the repetitive control scheme and the slidingmode technique are combined to provide both good transient performance and also complete insensitivity to periodic exogenous disturbances. However, the control scheme proposed in the study (Lu \& Wang, 2009) uses an integral sliding perturbation observer. This integral form would cause an undesired phenomenon of integrator windup, causing deterioration of system performance. Furthermore, in the sliding perturbation observer (Lu \& Wang, 2009), a switching gain has to be larger than the magnitude of unknown perturbation. However, it is demanding to decide the switching gain that is suitable to all operating conditions, and it involves trial and error to estimate the bounds for determining the switching gain.

In this study, a sliding repetitive controller with adaptive bound estimation is proposed. Compared with the approach in the study (Lu \& Wang, 2009), the proposed scheme contains a sliding disturbance observer of proportional form, which avoids the problem of integrator windup. Moreover, the proposed scheme adaptively estimates disturbance bounds and automatically decides the switching gain without the need of trial and error.

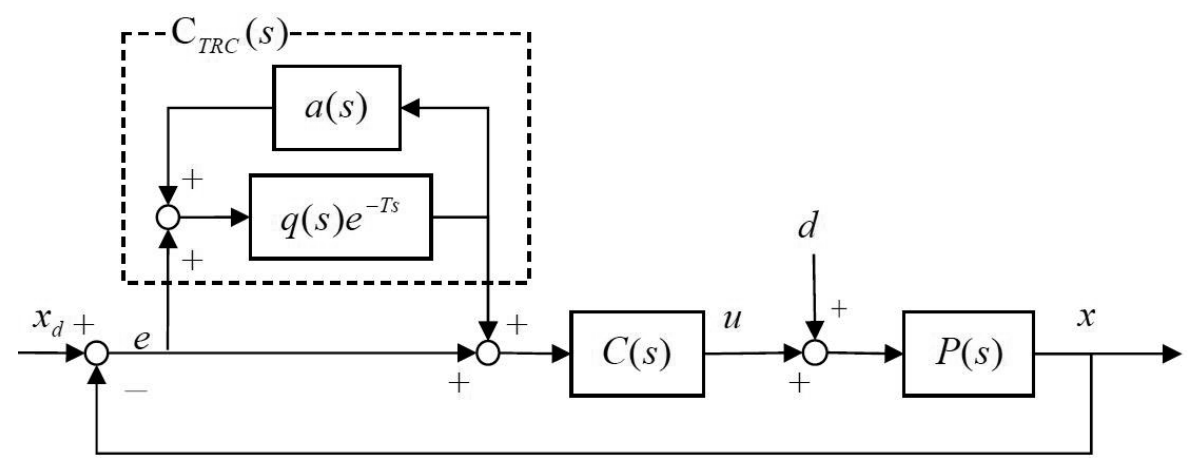

Fig. 1. A traditional repetitive control scheme.

\section{TRADITIONAL REPETITIVE CONTROLLER}

Traditional repetitive control (TRC) was introduced in the literature (Hara et al., 1988), whose structure is presented in Figure 1. Here, $P(s)$ represents the plant's transfer function, $x_{d}$ denotes a reference, $u$ and $x$ are, respectively, the plant's input and output, $e$ denotes a tracking error, $d$ is an unknown periodic exogenous disturbance, $C(s)$ is a nominal controller, $C_{T R C}(s)$ denotes the TRC, $q(s)$ denotes a low-pass filter, and $e^{-T s}$ represents a delay element, in which $T$ denotes the period of exogenous signals. Moreover, as it is in the literature (Doh et al., 2002), choose the filter, $a(s)$, to be equal to unity for comparison study in this work.

\section{A SLIDING REPETITIVE CONTROLLER \\ Plant Model and Controller Configuration}

A second-order plant is considered and described in compassion form: $\ddot{x}=-\mathbf{a}^{\mathbf{T}} \mathbf{x}+b u+\delta$, in which $\delta$ represents an unknown periodic input disturbance, the vector of state variables $\mathbf{x}=\left[\begin{array}{ll}x & \dot{x}\end{array}\right]^{T}=\left[\begin{array}{ll}x_{1} & x_{2}\end{array}\right]^{T}$, and $b$ and $\mathbf{a}=\left[\begin{array}{ll}a_{1} & a_{2}\end{array}\right]^{T}$ are system parameters. Define the output error vector, $\mathbf{e}=\left[\begin{array}{ll}e_{1} & e_{2}\end{array}\right]=\mathbf{x}-\mathbf{x}_{d}$, in which the reference vector $\mathbf{x}_{d}=\left[\begin{array}{ll}x_{d} & \dot{x}_{d}\end{array}\right]^{T}$. In this paper, $\mathbf{x}_{d}$ is supposed to be periodic but unavailable, and only the tracking error vector, $\mathbf{e}$, is available for feedback control. Because only the tracking error, rather than the reference, is measurable in some servo systems, such as disk drives. Rewrite the plant model as:

$$
\dot{e}_{2}=-\mathbf{a}^{\mathrm{T}} \mathbf{e}+b u+d,
$$

in which $d=-\mathbf{a x}_{d}-\ddot{x}_{d}+\delta$, representing the exogenous disturbance that could deteriorate output performance. 
The control scheme presented in this paper is described by

$$
u=u_{p a}+u_{d o}+u_{f f}^{i},
$$

where $u_{p a}$ is a nominal control, $u_{d o}$ is a compensation produced by a sliding disturbance observer, and $u_{f f}^{i}$ is a sliding repetitive control during the $i$-th period. The nominal control is designed using the pole-assignment technique and given by

$$
u_{p a}=\hat{b}^{-1}\left(\hat{\mathbf{a}}^{T} \mathbf{e}-\mathbf{c}^{T} \mathbf{e}\right)
$$

where $\hat{b}$ and $\mathbf{a}$ are respectively nominal values of $b$ and $\mathbf{a}$, and controller parameters, $\mathbf{c}=\left[\begin{array}{ll}c_{1} & c_{2}\end{array}\right]^{T}$, are chosen to specify a desired closed-loop characteristic equation:

$$
s^{2}+c_{2} s+c_{1}=0
$$

Combining Equations (2) and (3) with Equation (1) yields the closed-loop dynamics:

$$
\begin{aligned}
\dot{e}_{2} & =-\mathbf{c}^{\mathbf{T}} \mathbf{e}+b\left(u_{d o}+u_{f f}^{i}\right)+\left(b \hat{b}^{-1}-1\right)\left(-\mathbf{c}^{T} \mathbf{e}\right) \\
& +\left(b \hat{b}^{-1} \hat{\mathbf{a}}-\mathbf{a}\right)^{T} \mathbf{e}+d .
\end{aligned}
$$

The design objective is to devise $u_{d o}$ and $u_{f f}^{i}$ so that the following desired closed-loop dynamics is achieved:

$$
\dot{e}_{2}=-\mathbf{c}^{T} \mathbf{e},
$$

where the effects of parametric uncertainties and periodic exogenous disturbance should be suppressed.

\section{Disturbance Observation and Repetitive Control}

For disturbance observation and repetitive control, a sliding motion is produced inside the controller by introducing a virtual process given by

$$
\dot{\hat{e}}_{2}=-\mathbf{c}^{\mathbf{T}} \mathbf{e}+\hat{b} u_{d o}+u_{s w},
$$

where $\hat{e}_{2}$ denotes the state of this virtual process, and $u_{s w}$ represents a switching input to this process. Here, $u_{s w}$ is given by

$$
\begin{aligned}
& u_{s w}=\hat{b} u_{f f}^{i} \\
& +\left\{\Delta b\left|u_{f f}^{i}+u_{d o}\right|+\hat{b}^{-1} \Delta b\left|-\mathbf{c}^{T} \mathbf{e}\right|+\Delta d\right\} \operatorname{sgn}(\sigma),
\end{aligned}
$$

in which $\operatorname{sgn}(\cdot)$ represents the signum function, $\Delta b$ and $\Delta d$ respectively denote upper bounds on magnitudes of $b-\hat{b}$ and $d$, and $\sigma$ is referred to as a switching function defined by

$$
\sigma=e_{2}-\hat{e}_{2}
$$

It can be shown that the sliding condition, $\sigma \dot{\sigma}<0$ if $\sigma \neq 0$, can be satisfied if the switching gain is large enough. If the initial value of the virtual process's state variable is set to $\hat{e}_{2}(0)=e_{2}(0)$, giving $\sigma(0)=0$. Hence, the sliding mode is ensured all over a whole response (Lu \& Chen, 1995); that is,

$$
\sigma(t)=0
$$

for all time. It implies that $\dot{\sigma}(t)=0$ for all time, giving $\dot{\hat{e}}_{2}(t)=\dot{e}_{2}(t)$. Substituting Equation (7) into this relation yields 


$$
\dot{e}_{2}=-\mathbf{c}^{\mathbf{T}} \mathbf{e}+\hat{b} u_{d o}+u_{s w}
$$

To achieve the desired dynamics (6), let

$$
u_{d o}=-\hat{b}^{-1} \alpha(s) u_{s w} \text {. }
$$

in which $\alpha(s)$ denotes a low-pass filter to obtain the equivalent value of $u_{s w}$. Moreover, let the repetitive control law be

$$
u_{f f}^{i+1}(s)=\beta(s)\left(u_{f f}^{i}(s)-\hat{b}^{-1} u_{s w}^{i}(s)\right)
$$

in which ' $i$ ' denotes an associated variable in the $i$-th cycle, and $\beta(s)$ denotes a low-pass filter for enhancing system robustness. Figure 2 presents the configuration of the proposed system.

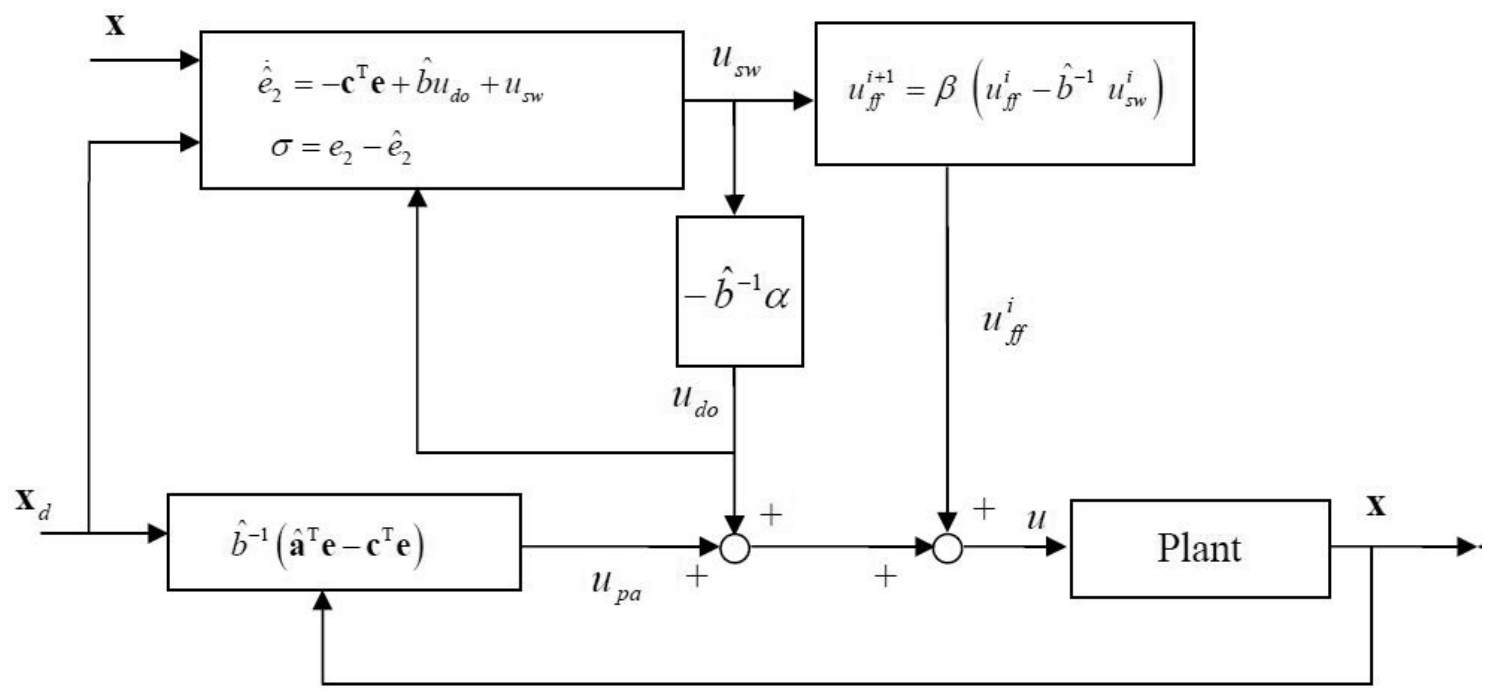

Fig. 2. A proposed repetitive control scheme.

\section{REALIZATION USING TRUNCATED FOURIER SERIES}

The proposed scheme, as shown in Figure 2, needs the implementation of the filter, $\beta(s)$, and also a lot of memories to store repetitive control efforts, $u_{f f}^{i}$. In this paper, an approach of truncated Fourier series is used to diminish these requirements.

Consider a complete orthogonal set, $\left\{\phi_{m}\right\}_{m=-\infty}^{m=\infty}$, which constitutes an orthogonal basis of the extended $L_{2}(\mathrm{Lu}, 2004)$. In this paper, $\phi_{k}=\cos (2 \pi k t / T)$ for $k=-1,-2, \ldots, \phi_{0}=1$, and $\phi_{k}=\sin (2 \pi k t / T)$ for $k=1,2, \ldots$. Define a variable representing local time, $\tau \in[0, T)$. The switching signal, $u_{s w}^{i}$, can be decomposed as

$$
u_{s w}^{i}(\tau+(i-1) T)=\sum_{m=-\infty}^{\infty} v_{m}^{i} \phi_{m}(\tau)
$$

in which the coefficients $v_{m}^{i}=\left\langle u_{s w}^{i}, \phi_{m}\right\rangle /\left\langle\phi_{m}, \phi_{m}\right\rangle$. Let the repetitive control, $u_{f f}^{i}$, be described by

$$
u_{f f}^{i}=\sum_{m=-M}^{M} \lambda_{m}^{i} \phi_{m}
$$

in which $M$ is a positive integer, and $\lambda_{m}^{i}$ are parameters to be adjusted. The updating rule for $\lambda_{m}^{i}$ is 


$$
\lambda_{m}^{i+1}=\lambda_{m}^{i}+\hat{b}^{-1} v_{m}^{i} \text { for }-M \leq m \leq M .
$$

The design parameter, $M$, should be chosen so that major components of the switching signal, $u_{s w}^{i}$, can be encompassed.

\section{ADAPTIVE BOUND ESTIMATION}

In Equation (8), the switching gain is formulated and needs to be estimated in order to successfully implement the proposed scheme. However, it is hard to estimate this switching gain due to the existence of unknown variables. An adaptive bound estimation law is thus required. Redefine the switching signal (8) as

$$
u_{s w}=K_{s i} \operatorname{sgn}(\sigma)
$$

in which $K_{s i}$ is the switching gain to be adapted. Subtracting Equation (7) from Equation (1) and combing Equations (2), (3) and (17) with the obtained equation, one has

$$
\dot{\sigma}+K_{s i} \operatorname{sgn}(\sigma)=\varphi
$$

in which $\varphi=(b-\hat{b}) u_{d o}+b u_{f f}^{i}+d+\left(b \hat{b}^{-1}-1\right)\left(-\mathbf{c}^{T} \mathbf{e}\right)+\left(b \hat{b}^{-1} \hat{\mathbf{a}}-\mathbf{a}\right)^{T} \mathbf{e}$. Define a variable

$$
\bar{K}_{s i}=|\varphi|+\xi,
$$

in which $\xi$ is a positive constant. Let $\kappa$ be a known constant satisfying $\left|\dot{\bar{K}}_{s i}\right|<\kappa$. The adaptive bound estimation law is described by (Lu \& Li, 2008)

$$
\dot{K}_{s i}= \begin{cases}-\kappa, & \text { if }\left|\dot{\sigma}+K_{s i} \operatorname{sgn}(\sigma)\right|+\xi<K_{s i}, \\ \gamma|\sigma|+\kappa, & \text { otherwise }\end{cases}
$$

where $\gamma$ is a positive constant. If $\left|\dot{\sigma}+K_{s i} \operatorname{sgn}(\sigma)\right|+\xi \geq K_{s i}$, it implies that the present switching gain is too small and should be increased according to $\dot{K}_{s i}=\gamma|\sigma|+\kappa$. On the contrary, when $\left|\dot{\sigma}+K_{s i} \operatorname{sgn}(\sigma)\right|+\xi<K_{s i}$, the switching gain can be decreased by $\dot{K}_{s i}=-\kappa$.

\section{EXPERIMENTAL STUDY Plant under Study}

In this study, the tracking actuator of a disk drive is the plant under study. For more details on this experimental system, shown in Figure 3, please refer to the study (Lu \& Li, 2008). Figure 4 depicts the measured frequency response of the tracking actuator using the swept-sine method by a dynamic signal analyzer. This figure also shows a curvefitting result for obtaining a mathematic model. Here, the tracking actuator is modeled as $\ddot{e}=-2 \zeta \omega_{n} \dot{e}-\omega_{n}^{2} e+b u+d$ with identified parameter values: $\hat{b}=1.543 \times 10^{9}, \omega_{n}=708.80 \mathrm{rad} / \mathrm{s}$, and $\zeta=0.05(\mathrm{Lu}, 2010)$. In this study, the sampling frequency of the tracking servo is $195.31 \mathrm{kHz}$. 
Optical Disk

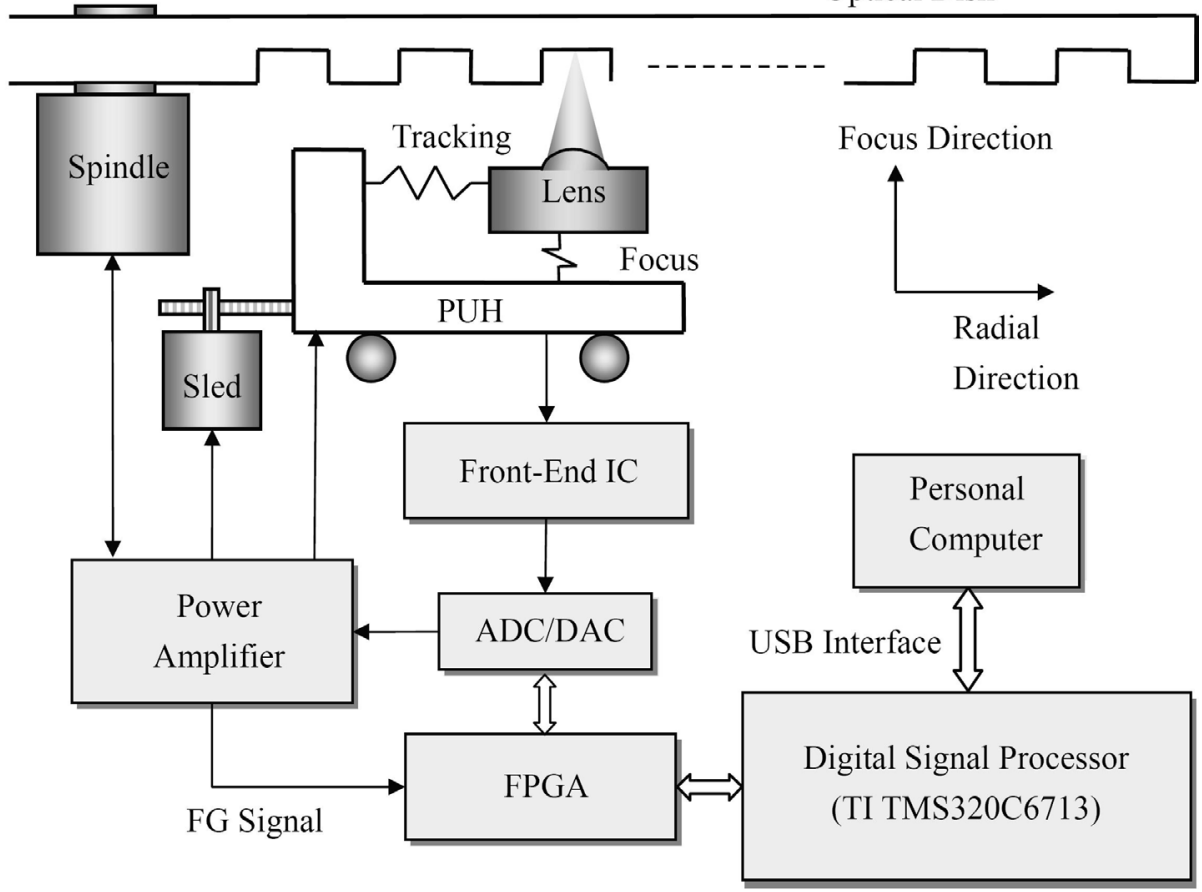

Fig. 3. Schematic of the experimental system ( $\mathrm{Lu} \& \mathrm{Li}, 2008)$.
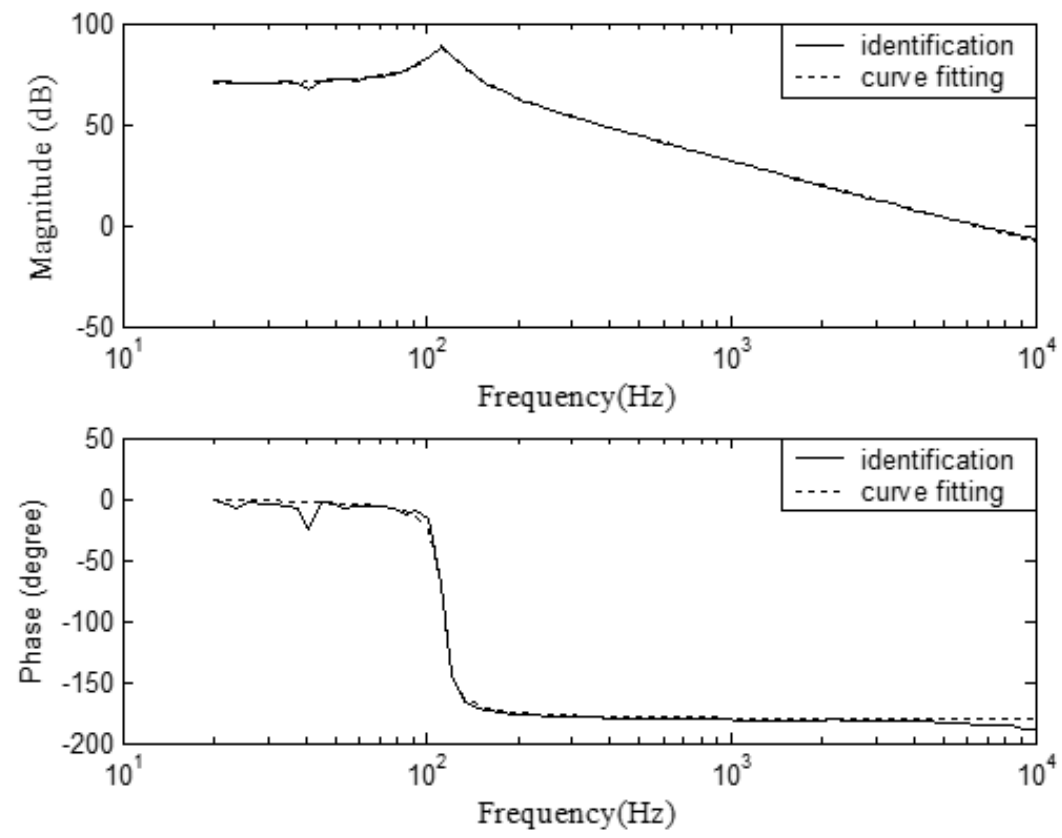

Fig. 4. Measured response of the experimental plant and its curve fitting result. 


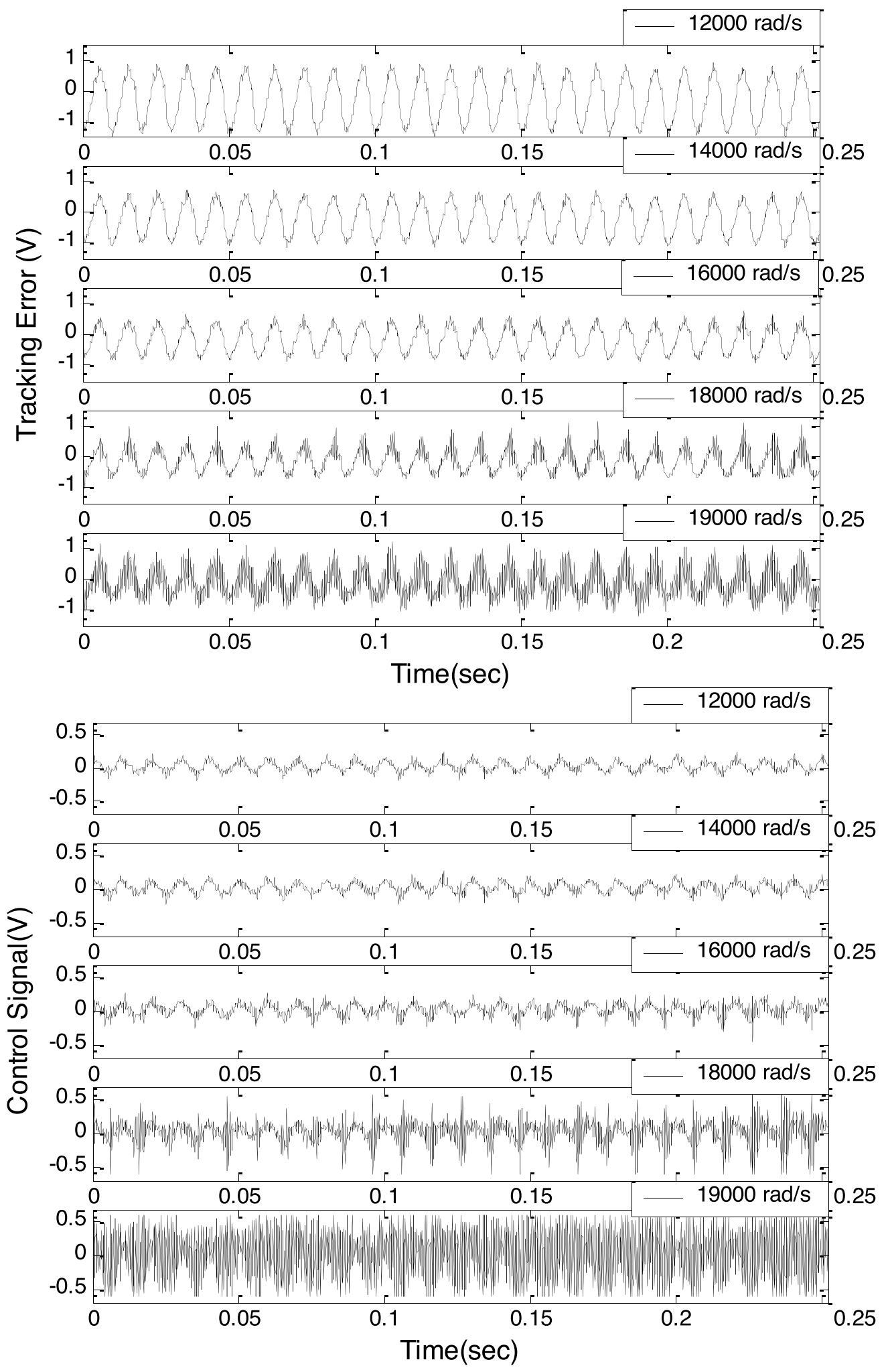

Fig. 5. Tracking responses using the nominal controller only. 
To evaluate efficacy of the suggested scheme on the track-following system, a TEAC DVD-ROM disk with a radial eccentricity of $\pm 100 \mu \mathrm{m}$ is used. In succeeding experiments, the spindle motor's velocity, which is also the disk's rotating rate, is $6000 \mathrm{rpm}$, implying that the periodic exogenous signal of this track-following system has a main component with a frequency of $100 \mathrm{~Hz}$.

When only the nominal controller is used, that is, $u=u_{p a}$, Figure 5 presents the tracking responses with different nominal closed-loop poles. It is seen that the tracking error can be diminished by increasing the feedback gains. However, the control efforts become chattering with the increase in feedback gains. Hence, the nominal controller only is unable to cope with the exogenous disturbance effectively.

\section{Experimental Results with a Fixed Switching Gain}

The presented control consists of the following three control efforts: the nominal control, $u_{p a}$; the sliding disturbance compensation, $u_{d o}$; and the sliding repetitive control, $u_{f f}^{i}$. For the nominal control, the poles of the desired closedloop dynamics are placed at -12000 with a multiplicity of two in subsequent experiments. Concerning the sliding disturbance observer, the corner frequency of the filter, $\alpha(s)$, is set to $6000 \mathrm{rad} / \mathrm{s}$, and this filter has unity $d c$ gain. As for sliding repetitive control, the low-pass filter, $\beta(s)$, also has unity $d c$ gain with a corner frequency of $6000 \mathrm{rad} / \mathrm{s}$. For implementation using the truncated Fourier series, choose $M=1$. The switching gain is fixed and set to $0.17 \hat{b}$.

To further evaluate the track-following capability, a step input having a step size of $0.1 \mathrm{~V}$ is applied to the plant's input at $t=0.1 \mathrm{~s}$. Figure 6 shows tracking responses using three different control laws: $u=u_{p a}+u_{d o} ; u=u_{p a}+u_{f f}^{i}$; and $u=u_{p a}+u_{d o}+u_{f f}^{i}$. It is seen that the tracking output contains significant periodic errors without using $u_{f f}^{i}$. Moreover, both the transient performance and also the response to the input disturbance are poor without the aid of $u_{d o}$. On the other hand, the proposed law, $u=u_{p a}+u_{d o}+u_{f f}^{i}$, produces the best performance among these three control laws.

As a comparison study, the traditional repetitive controller (TRC) is used, in which the filter, $q(s)$, has a $d c$ gain of unity with a cutoff frequency of $10000 \mathrm{rad} / \mathrm{s}$. Figure 7 shows tracking responses using the TRC and two implementations of the proposed scheme. One implementation makes use of a low-pass filter, $\beta(s)$, while the other uses the truncated Fourier series. From Figure 7, it is seen that without the aid of a sliding disturbance observer, the transient performance of the TRC is worse than that of the proposed scheme. Moreover, the TRC cannot instantly counteract the additional step disturbance and needs one extra cycle to react to this input disturbance. The two implementations of the proposed scheme produce similar output responses. However, the implementation using the truncated Fourier series gives less chatter in the control signal than that using the low-pass filter. Because the implementation using the truncated Fourier series can selectively suppress certain frequency components of the tracking errors without exciting other frequency components. 

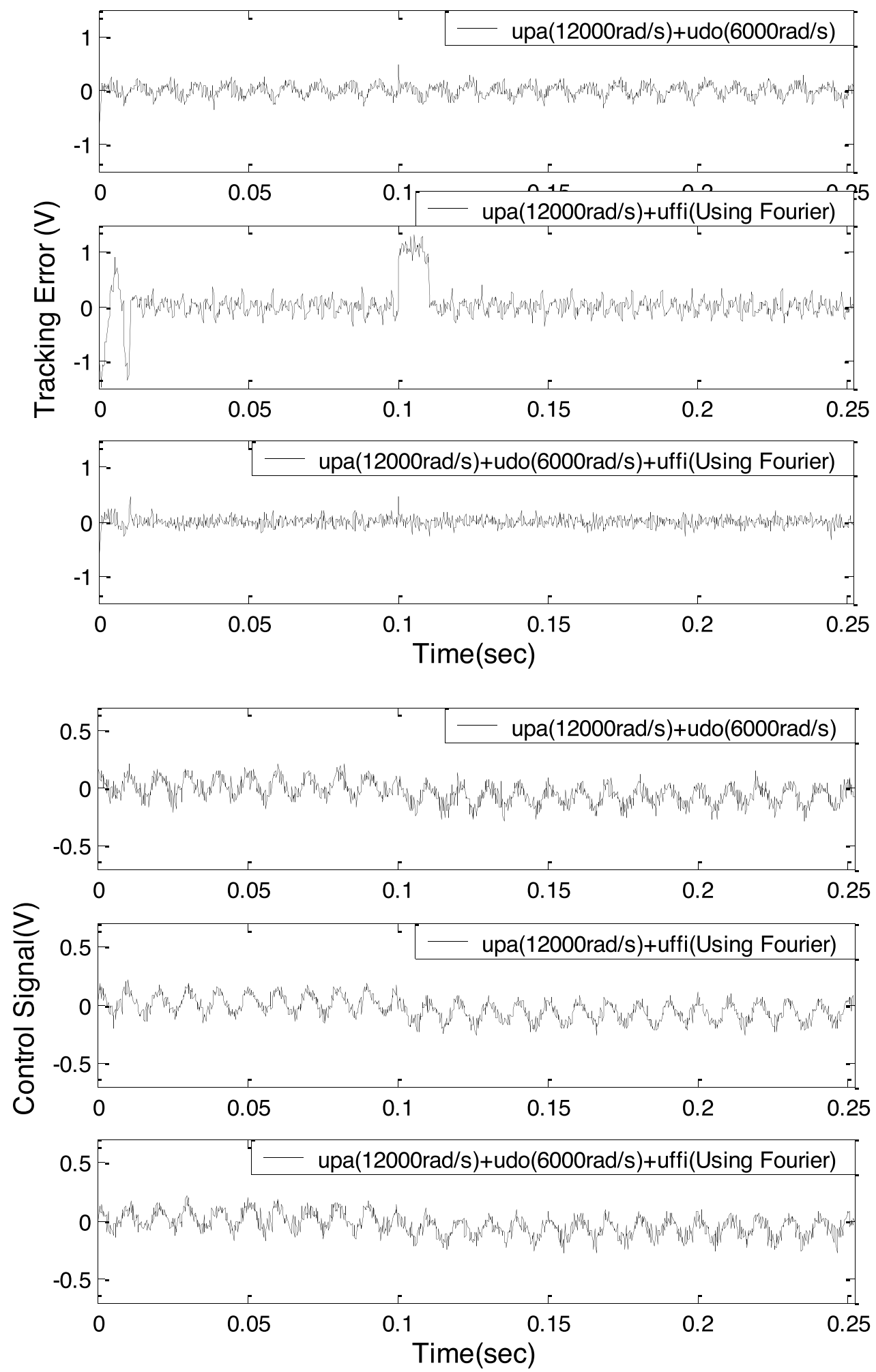

Fig. 6. Tracking responses subject to an additional input disturbance. 

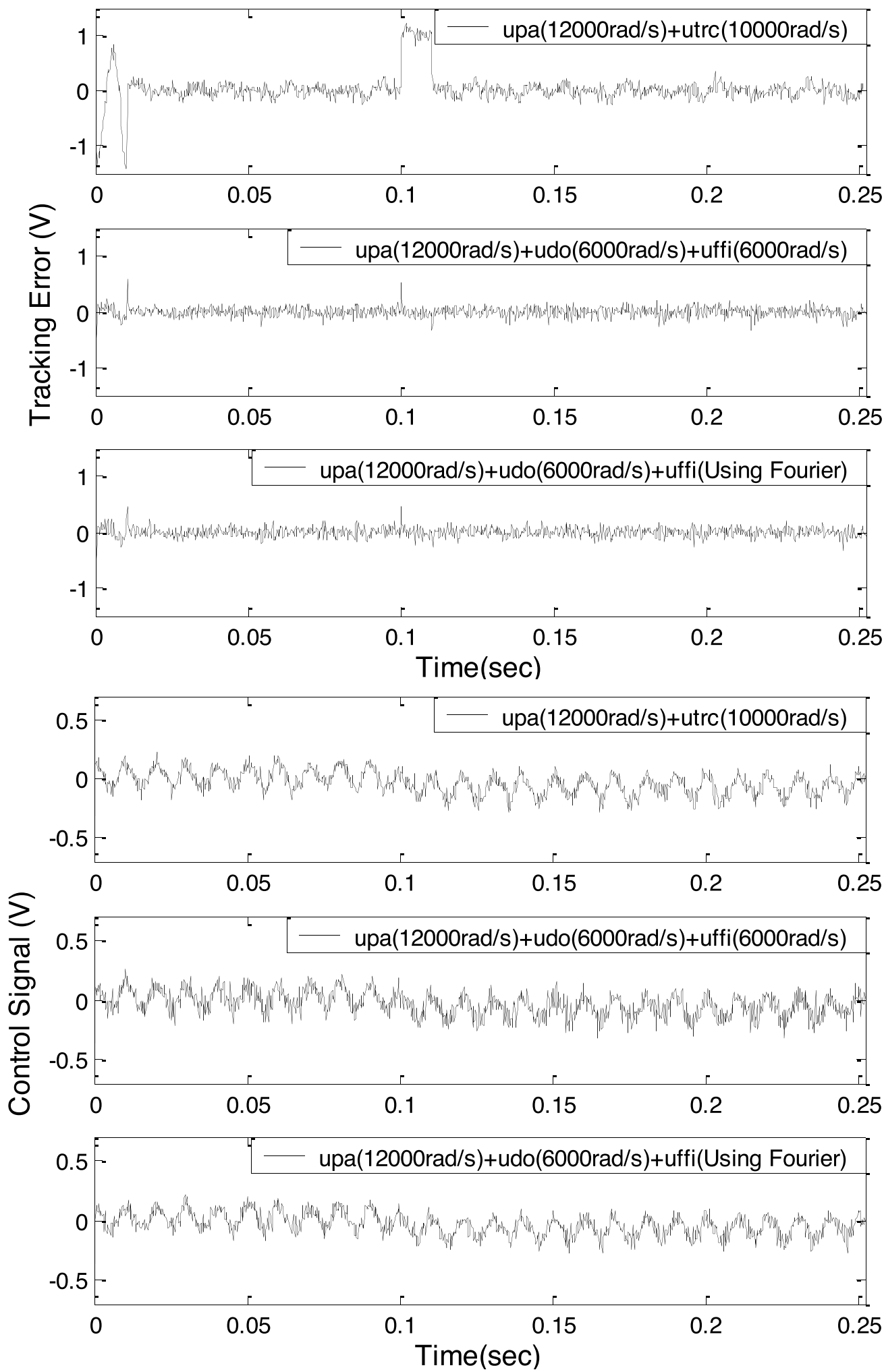

Fig. 7. Tracking responses subject to an additional input disturbance using a traditional repetitive controller (TRC). 

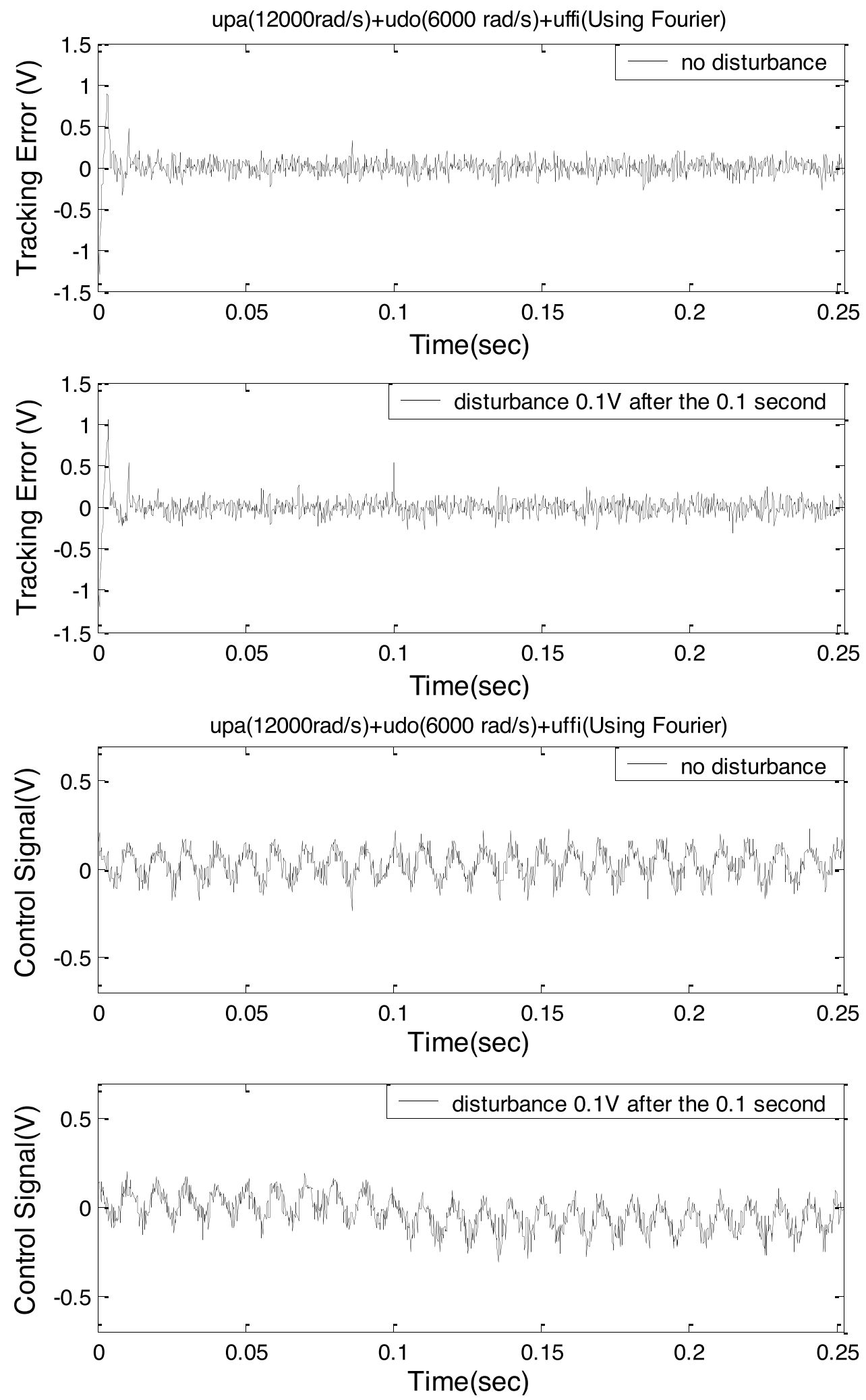

Fig. 8. Tracking responses with adaptive bound estimation and with/without an additional input disturbance. 


\section{Experimental Results with a Variable Switching Gain}

In the previous experiments, the switching gain is a constant equal to $0.17 \hat{b}$. The switching gain has to be larger than the maximum of system perturbation, so that a sliding motion can be ensured to exist for both disturbance observation and repetitive control. However, this fixed switching gain may not be able to guarantee the presence of a sliding motion in other operating conditions of the disk drive.

In the following experiments, the switching gain is adapted according the bound estimation law (20) with parameters: $\kappa=20 \hat{b}, \gamma=5 \hat{b} \times 10^{-13}$, and $\xi=5 \times 10^{-6}$. Figure 8 shows tracking responses with and without an additional step disturbance added to the plant input at $t=0.1 \mathrm{~s}$. It is seen that the adaptation mechanism works well in both cases with and without an additional input disturbance. Moreover, the chatter levels of the control signals resulting from the adaptation mechanism are comparable to those with a fixed switching gain. Figure 9 shows adapted switching gains with and without the additional input disturbance. It is seen that after the step input is applied at $t=0.1 \mathrm{~s}$, the adaptation mechanism increases the switching gain to counteract this additional disturbance. Afterwards the adaptation mechanism decreases the switching gain in order to alleviate the chattering phenomenon.
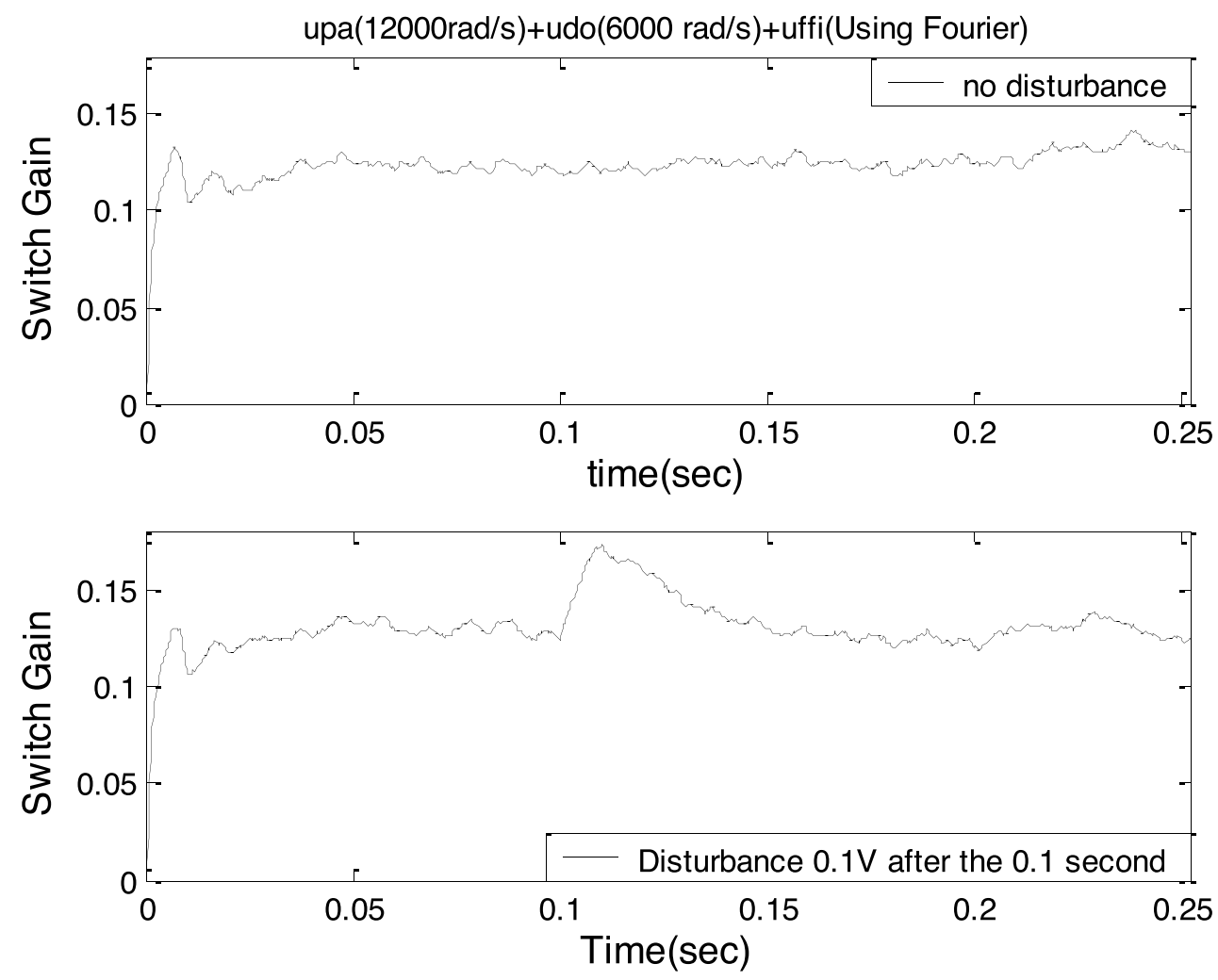

Fig. 9. Adapted switching gains with/without an additional input disturbance.

\section{CONCLUSIONS}

In this paper, a scheme of sliding repetitive control is presented for motion systems suffering from periodic exogenous signals. In contrast to the traditional repetitive controller, the proposed sliding repetitive controller is augmented with a sliding disturbance observer, providing improved transient performance of a control system. The sliding disturbance observer is of a proportional type, avoiding the integral windup problem. Moreover, the switching gain that is difficult to determine in practice can be adaptively tuned using the proposed bound estimation law. The proposed scheme was practically implemented on a disk drive's track-following system and compared with the 
traditional repetitive control scheme. Experimental results show the superiority of the proposed controller to the traditional repetitive controller. Moreover, the presented bound estimation law can practically adjust the switching gain according to actual operating conditions.

\section{ACKNOWLEDGMENT}

Research is supported by Ministry of Science and Technology, Taiwan, under Grants No. MOST 108-2221-E003-023.

\section{REFERENCES}

Alsubaie, M.A. \& Rogers E. 2019. State feedback repetitive control design: robustness and load disturbance conditions. Journal of Engineering Research, 7: 165-177. https://kuwaitjournals.org/jer/index.php/JER/article/view/7004

Chen, Y., Moore, K.L., Yu, J. \& Zhang, T. 2008. Iterative learning control and repetitive control in hard disk drive industry - a tutorial. International Journal of Adaptive Control and Signal Processing, 22: 325-343. https://doi.org/10.1002/acs.1003

Doh, T.Y., Ryoo, J.R. \& Chung, M.J. 2002. Repetitive control for the track-following servo system of an optical disk drive. Proceedings of the 7th International Workshop on Advanced Motion Control, 176-181. DOI: 10.1109/AMC.2002.1026912

Fateh, M.M., Tehrani, H.A. \& Karbassi, S.M. 2013. Repetitive control of electrically driven robot manipulators. International Journal of Systems Science, 44: 775-785. https://doi.org/10.1080/00207721.2011.625478

Gu, D.W., Petkov, P.H. \& Konstantinov, M.M. 2014. Robust Control Design with MATLAB. Springer, London.

Hara, S., Yamamoto, Y., Omata, T. \& Nakano, M. 1988. Repetitive control system: a new type servo system for periodic exogenous signals. IEEE Transactions on Automatic Control, 33: 659-668. DOI: 10.1109/9.1274

Hillerstrom, G. 1996. Adaptive suppression of vibrations - a repetitive control approach. IEEE Transactions on Control Systems Technology, 4: 72-78. DOI: 10.1109/87.481769

Jiangand X. \& Hong, H. 2019. Analysis and design of the plug-in type repetitive control system based on steady-state residual convergence ratio. International Journal of Advanced Robotic Systems, 16: 1-12. https://doi.org/10.1177/1729881419850977

Kara, T. \& Mary, A.H. 2018. Robust trajectory tracking control of robotic manipulators based on model-free PID-SMC approach. Journal of Engineering Research, 6: 170-188. https://kuwaitjournals.org/jer/index.php/JER/article/view/2735

Lu, Y.S. 2004. Orthogonal-function-based repetitive control with a composite sliding perturbation estimator. Journal of the CSME, 25: 515-525.

Lu Y.S. 2010. Internal model control of lightly damped systems subject to periodic exogenous signals. IEEE Transactions on Control Systems Technology, 18: 699-704. DOI: 10.1109/TCST.2009.2023672

Lu Y.S. \& Chen, J.S. 1995. Design of a perturbation estimator using the theory of variable-structure systems and its application to magnetic suspension systems. IEEE Transactions on Industrial Electronics, 42: 281-289. DOI: 10.1109/41.382139

Lu Y.S. \& Li, Y.T. 2008. Design of a sliding perturbation estimator with bound estimation. Proceedings of the IEEE VSS'08 308-313. DOI: $10.1109 /$ VSS.2008.4570726

Lu, Y.S. \& Wang, X.W. 2009. Sliding-mode repetitive learning control with integral sliding-mode perturbation compensation. ISA Transactions, 48: 156-165. https://doi.org/10.1016/j.isatra.2008.10.013

Ma, G., Liu, X. \& Pagilla, P.R. 2019. Robust repetitive control of semi-Markovian jump systems. International Journal of Systems Science, 50: 116-129. https://doi.org/10.1080/00207721.2018.1543480

Oliveira I.G. de \& Lages, W.F. 2016. Repetitive control applied to robot manipulators. Proceedings of the 2016 IEEE 21st International Conference on Emerging Technologies and Factory Automation, 1-8. DOI: 10.1109/ETFA.2016.7733549

Xu, X., Chen, S. \& Liu, J. 2017. Elimination of harmonic force and torque in active magnetic bearing systems with repetitive control and notch filters. Sensors (Basel) 17: article no. 763. https://doi.org/10.3390/s17040763

Yang, Y., Huang, D., \& Dong, X. 2018. Robust repetitive learning control of lower limb exoskeleton with hybrid electro-hydraulic system. Proceedings of the 2018 IEEE 7th Data Driven Control and Learning Systems Conference, 718-723. DOI: 10.1109/ DDCLS.2018.8516005 